\title{
LECS, LOCAL MIXERS, TOPOLOGICAL GROUPS AND SPECIAL PRODUCTS
}

\author{
CARLOS R. BORGES
}

(Received 18 December 1986)

Communicated by J. H. Rubinstein

\begin{abstract}
We prove that every (locally) contractible topological group is (L)EC and apply these results to homeomorphism groups, free topological groups, reduced products and symmetric products. Our main results are: The free topological group of a $\theta$-contractible space is equiconnected. A paracompact and weakly locally contractible space is locally equiconnected if and only if it has a local mixer. There exist compact metric contractible spaces $X$ whose reduced (symmetric) products are not retracts of the Graev free topological groups $F(X)(A(X))$ (thus correcting results we published ibidem).
\end{abstract}

1980 Mathematics subject classification (Amer. Math. Soc.): primary 54 C 55; secondary 22 A $05,57 \mathrm{~S} 05$.

Keywords and phrases: $\theta$-contractible, free topological group, reduced product, symmetric product, (L)EC, (local) mixers, homeomorphism groups.

\section{Introduction}

Let us recall that a space $X$ is LEC (that is, locally equiconnected) if there exists a continuous function $\lambda: U \times I \rightarrow X$, where $U$ is a neighbourhood of the diagonal of $X$, such that $\lambda(x, y, 0)=x, \lambda(x, y, 1)=y$ and $\lambda(x, x, t)=x$. $X$ is EC (that is equiconnected) if $U=X \times X$.

Letting $\Delta^{*}=\{(x, x, y),(x, y, x),(y, x, x) \mid x, y \in X\}$ a local mixer for $X$ is a continuous map $\mu: U \rightarrow X$ of a neighbourhood $U$ of $\Delta^{*}$ in $X \times X \times X$ which satisfies the following condition: for each $x \in X$ and neighbourhood $V$ of $x$, there

(C) 1988 Australian Mathematical Society $0263-6115 / 88 \$ A 2.00+0.00$ 
exists a neighbourhood $W$ of $x$ such that

$$
X \times W \times W \cup W \times X \times W \cup E \times W \times X \subset \mu^{-1}(V) .
$$

If $U=X \times X \times X$ then we call $\mu$ a mixer for $X$.

It is well known that ANR (metrizable) spaces are LEC (see [6]) and it is conjectured that (metrizable) LEC spaces are ANE (metrizable) (see [1] and [2] for partial answers to this conjecture).

In [13], a space $X$ is called semi-locally contractible provided that it has an open cover $\left\{U_{\alpha} \mid \alpha \in \Lambda\right\}$, a family $\left\{\left\{a_{\alpha}\right\} \mid \alpha \in \Lambda\right\}$ of singleton subsets and a family $\left\{p_{\alpha}: U_{\alpha} \times I \rightarrow X \mid \alpha \in \Lambda\right\}$ of continuous functions such that each $p_{\alpha}(u, 0)=u$ and $p_{\alpha}(u, 1)=a_{\alpha}$, for all $u \in U_{\alpha}$. If each $a_{\alpha} \in U_{\alpha}$ then $X$ is said to be weakly locally contractible. For convenience, let us say that each $U_{\alpha}$ is contractible over $X$ (to $a_{\alpha}$ ) and $p_{\alpha}$ deforms $U_{\alpha}\left(\right.$ to $\left.a_{\alpha}\right)$ over $X$. Fortunately, the concept of semi-local contractiblity is equivalent to that of weak local contractibility (see Lemma 6.1 in the Appendix).

Sakai [13] proves that a metrizable space is (L)EC if and only if it is (semilocally) contractible and has a (local) mixer. Our Corollary 3.2 generalizes this result to paracompact spaces.

\section{LEC groups}

We prove that weak locally contractible topological groups are LEC. Let us recall that if $X$ is a topological space, $W$ is a neighbourhood of the diagonal of $X$ and $z \in X$, then $W[z]=\{x \in X \mid(z, x) \in W\}$.

LEMMA 2.1. Let $H$ be a topological group and $P$ an open symmetric neighbourhood of its unit element. Then there exists an open symmetric neighbourhood $W$ of the diagonal of $H$ such that $W[f] \subset f P$, for each $f \in H$.

ProOF. Let $W=\left\{(f, g) \in H \times H \mid f^{-1} g \in P\right\}$ and note that $W$ is an open symmetric neighbourhood of the diagonal of $H$ (note that $\mu: H \times H \rightarrow H$, defined by $\mu(f, g)=f^{-1} g$, is continuous and $\left.W=\mu^{-1}(P)\right)$. We also get that, for each $f \in H, W[f] \subset f P$, because $(f, g) \in W$ if and only if $f^{-1} g \in P$ if and only if $g \in f P$. This completes the proof.

Proposition 2.2. Let $H$ be a topological group, $P$ an open symmetric neighbourhood of its unit element $e$ and $\psi: P \times I \rightarrow H$ a continuous function such that $\psi(h, 0)=h, \psi(h, 1)=e$ and $\psi(e, t)=e$, for all $t \in I$ and $h \in P$. Then $H$ is $L E C$. 
ProOF. By Lemma 2.1, let $W$ be a symmetric neighbourhood of the diagonal such that $W[f] \subset f p$, for all $f \in H$. Define $\lambda: W \times I \rightarrow H$ by

$$
\lambda(g, f, t)=f \psi\left(f^{-1} g, t\right) .
$$

(Note that $\lambda$ is well defined, since $(g, f) \in W \Leftrightarrow(f, g) \in W \Rightarrow g \in W[f] \subset$ $f P \Rightarrow f^{-1} g \in P$.) Then $\lambda(g, f, 0)=f \psi\left(f^{-1} g, 0\right)=f f^{-1} g=g, \lambda(g, f, 1)=$ $f \psi\left(f^{-1} g, 1\right)=f e=f$, and $\lambda(g, g, t)=g \psi\left(g^{-1} g, t\right)=g \psi(e, t)=g e=g$. So we need only prove that $\lambda$ is continuous, to complete the proof: Let $\left\{\left(g_{\nu}, f_{\nu}, t_{\nu}\right)\right\}$ be a net in $W \times I$ which converges to (g.f.t). Using the continuity of the inverse and multiplication maps for the topological group $H$, we then get that $\lim _{\nu} f_{\nu}^{-1}=f^{-1}$ and $\lim _{\nu} f_{\nu}^{-1} g_{\nu}=f^{-1} g$; so $\lim _{\nu} \psi\left(f_{\nu}^{-1} g_{\nu}, t_{\nu}\right)=\psi\left(f^{-1} g, t\right)$ and $\lim _{\nu} f_{\nu} \psi\left(f_{\nu}^{-1} g_{\nu}, t_{\nu}\right)=f \psi\left(f^{-1} g, t\right)$; equivalently, $\lim _{\nu} \lambda\left(g_{\nu}, f_{\nu}, t_{\nu}\right)=\lambda(g, f, t)$, which proves that $\lambda$ is continuous.

THEOREM 2.3. Let $H$ be a topological group. $H$ is LEC if and only if some nonempty open subset $U$ of $H$ is contractible over $H$ to some $g \in H$.

PROOF. The "if" part follows immediately from Proposition 6.2 in the Appendix and Proposition 2.2.

The "only if" part: Let $D$ be a neighbourhood of the diagonal of $X$ and $\lambda: D \times I \rightarrow X$ a continuous map such that $\lambda(f, g, 0)=f, \lambda(f, g, 1)=g$ and $\lambda(f, f, t)=f$. Pick a neighbourhood $U$ of $e$ such that $U \times U \subset D$ and define $\mu: U \times I \rightarrow H$ by $\mu(u, t)=\lambda(u, e, t)$; note that $\mu$ is continuous, $\mu(u, 0)=u$ and $\mu(u, 1)=e$, which shows that $U$ is contractible over $H$. This completes the proof.

The preceding results prove that many homeomorphism groups are LEC. Henceforth, if $M$ is a compact $n$-manifold, or a manifold which is homeomorphic to the interior of a compact $n$-manifold, and $(M, N)$ is a proper manifold pair, let $\mathcal{H}(M)\left(H(M, N) ; H_{1}(M, N)\right)$ denote the group of homeomorphisms of $M$ (which are invariant on $N$; the identity on $N$ ) with the compact-open topology.

THEOREM 2.4. The homeomorphism groups $\mathcal{H}(M), \mathcal{H}(M, N)$ and $\mathcal{H}_{1}(M, N)$ are $L E C$.

ProOF. From Corollaries 1.4 and 6.1 of [9] and Theorem 2.3, we get that $\mathcal{H}(M)$ is LEC. From Corollary 7.3 of [9] and Theorem 2.3, we get that $\mathcal{H}(M, N)$ and $\mathcal{H}_{1}(M, N)$ are LEC. 


\section{Equivalence of LEC and local mixers}

The following results generalize Theorem I and the main Theorem of Sakai [13] to paracompact spaces.

THEOREM 3.1. Let $(X, \tau)$ be a paracompact space. If $X$ is $(L) E C$ then $X$ has a (local) mixer.

ProOF. (We only need to make some additions to the Proof of Theorem I of [13].) Let $U$ be an open neighbourhood of the diagonal in $X^{2}$ and $\lambda: U \times$ $I \rightarrow X$ a local equiconnecting function. For each $a \in X$, let $U_{a}^{\prime}$ and $U_{a}^{\prime \prime}$ be open neighbourhoods of $a$ in $X$ such that $U_{a}^{\prime} \times U_{a}^{\prime} \subset U$ and $\lambda\left(U_{a}^{\prime \prime} \times U_{a}^{\prime \prime} \times\right.$ $I) \subset U_{a}^{\prime}$. Since $X$ is paracompact, let $d$ be a pseudometric for $X$ such that (the topology generated by $d) \tau_{d} \subset \tau$ and $\{\bar{B}(a, 1) \mid a \in X\}$ refines $\left\{U_{a}^{\prime \prime} \mid a \in X\right\}$, where $\bar{B}(a, 1)=\{x \in X \mid d(a, x) \leq 1\}$ (this can be done-see Lemma 38.1 of [14]). Next, define a pseudometric $d^{*}$ on $X \times X \times X$ by $d^{*}\left((x, y, z),\left(x^{\prime}, y^{\prime}, z^{\prime}\right)\right)=$ $\max \left\{d\left(x, x^{\prime}\right), d\left(y, y^{\prime}\right), d\left(z, z^{\prime}\right)\right\}$ and note that $\tau_{d^{*}} \subset \tau^{3}$ (the product topology on $X \times X \times X$ generated by $\tau)$.

The remainder of this proof is the same as that of Theorem I of [13], if one uses the topologies $\tau_{d}$ and $\tau_{d^{*}}$, except for letting $W$ be a $\tau$-neighbourhood of $a$ and picking a $\tau$-neighbourhood $W^{\prime \prime}$ of a such that $W^{\prime \prime} \subset \bar{B}(a, 1)$, keeping in mind that $\left(\tau_{d^{-}}\right) \tau_{d^{*}}$-open or closed sets are also $\left(\tau_{-}\right) \tau^{3}$-open or closed sets.

COROLLARY 3.2. Let $X$ be a paracompact space which is weak locally contractible. Then $X$ is LEC if and only if $X$ has a local mixer. If $X$ is also contractible then $X$ is $E C$ if and only if $X$ has a mixer.

ProOF. Immediate from Theorem 1.3 and 1.5 of [3] and Theorem 3.1.

\section{EC Graev groups}

Concerning Graev free topological groups, throughout we will use the terminology of [4]. For the sake of brevity, we say that the space $X$ is $\theta$-contractible if $\theta \in X$ and there exsits a homotopy $h: X \times I \rightarrow X$ such that $h(x, 0)=x, h(x, 1)=$ $\theta=h(\theta, t)$. (Let us call $h$ a $\theta$-homotopy.) It is possible for a compact metric space $X$ to be contractible to a point $\theta \in X$ but not $\theta$-contractible (see Example $6.4)$.

Even though we get very general conditions for the Graev free groups $(A(X), \mathcal{G})$ of a Tychonoff space $X$ to be EC, we cannot find satisfactory conditions for those groups to be LEC. 
THEOREM 4.1. If $X$ is a $\theta$-contractible Tychonoff space then $(A(X), \mathcal{G})$ and $(F(X), \mathcal{G})$ are $E C$.

Proof. By the Proposition on page 2 of [12], we get that $(A(X), \mathcal{G})$ and $(F(X), \mathcal{G})$ are contractible. (The above mentioned proposition is proved for abelian groups $A(X), \mathcal{G})$, but it is clearly valid for $(F(X), \mathcal{G})$.) Therefore, by Theorem 2.3, $(A(X), \mathcal{G})$ and $(F(X), \mathcal{G})$ are contractible LEC spaces, which implies that they are EC.

QUESTION 4.2. If $X$ is a Tychonoff locally contractible space are $(F(X), \mathcal{G})$ and $(A(X), \mathcal{G})$ LEC?

\section{Symmetric and reduced products}

In contrast to Theorem 4.1, there exists a compact subspace $H$ of the euclidean plane whose reduced product $H_{\infty}$ and symmetric product $S P^{\infty} H$ are not EC. Before establishing this fact, let us recall that a space $X$ is an $h$-space relative to $\theta \in X$ provided that there exists a continuous map $\mu: X \times X \rightarrow X$ such that $\mu(x, \theta)=\mu(\theta, x)=x$, for all $x \in X$. We will call $X$ a symmetric $h$-space relative to $\theta \in X$ if also $\mu(a, b)=\mu(b, a)$, for all $a, b \in X$.

Let $H$ be the "fan" subspace of the euclidean plane $E^{2}$ defined by $H=$ $\{(0, y) \mid 0 \leq y \leq 1\} \cup\left\{(x, y) \in E^{2}|| x \mid \leq 1\right.$ and $y=k x$ for some $\left.k=0,1, \ldots\right\}$.

LEMMA 5.1. The space $H$ is compact metric and a symmetric $h$-space.

Proof. Clearly $H$ is compact metric. So, letting $\overline{a b}$ denote the line segment joining $a$ to $b$ in $E^{2}$, let us define a function $\mu: H \times H \rightarrow H$ by

$$
\mu(a, b)=\left\{\begin{array}{lll}
p \in \overline{\theta a}, & \text { with }|p|=|a|-|b|, & \text { if }|a|>|b|, \\
q \in \overline{\theta b}, & \text { with }|q|=|b|-|a|, & \text { if }|b|>|a|, \\
\theta, & \text { if }|a|=|b|,
\end{array}\right.
$$

where $\theta$ denotes the origin of $E^{2}$. It is easily seen that $\mu$ is continuous. Clearly $\mu(a, \theta)=a=\mu(\theta, a)$; therefore $H$ is a symmetric $h$-space.

PROPOSITION 5.2. The reduced product $H_{\infty}$ and the symmetric product $S P^{\infty} H$ are $\theta$-contractible but they are not $E C$.

Proor. (We deal only with $H_{\infty}$, since the proof for $S P^{\infty} H$ is essentially the same.) Clearly $H$ is $\theta$-contractible (define $\mu: H \times I \rightarrow H$ by $\mu(x, t)=t x$ ). Therefore, we easily get that $H_{\infty}$ is $\theta$-contractible, by multiplying the homotopy $\mu$. Therefore, $(F(H), \mathcal{G})$ is EC, by Theorem 4.1. Now, if $H_{\infty}$ were a continuous 
retract of $(F(H), \mathcal{G})$, then $H$ would also be a continuous retract of $(F(H), \mathcal{G})$, by Theorem 1.8 of [11] (clearly, this result remains valid for symmetric $h$-spaces and symmetric products); consequently, $H$ would be $\mathrm{EC}$, a contradiction ( $H$ is not even locally connected).

Proposition 5.2 shows that Theorems 2.6, 2.7, and 3.2 of [5] are false. (It is noteworthy that the error lies in the diagram of Theorem 2.6 of [5]. By starting with $\left(x_{1}, \ldots, x_{i}, x_{i}^{-1}, \ldots, x_{n}\right) \in\left(A_{1}(X)\right)^{n}$, one immediately sees that it is not commutative.)

\section{Appendix}

The first two results should be folklore, but we cannot find them in the literature. The last result, which appears to be new, should prove very useful for a variety of problems on the extension of continuous functions.

Lemma 6.1. Let $X$ be a space, $q \in U \subset X$ and $p \in X$. If there exists a continuous map $\mu: U \times I \rightarrow X$ such that $\mu(u, 0)=u$ and $\mu(u, 1) p$ then $U$ is contractible over $X$ to $q$.

Proof. Let $\alpha=\mu \mid\{q\} \times I$ and define $\psi: U \times I \rightarrow X$ by

$$
\psi(u, t)= \begin{cases}\mu(u .2 t), & 0 \leq t \leq 1 / 2 \\ \alpha(2-2 t), & 1 / 2 \leq t \leq 1\end{cases}
$$

It is easily seen that $\psi$ satisfies all requirements.

PROPOSITION 6.2. Let $H$ be a topological group and $W$ a nonempty open subset of $H$ which is contractible over $H$ to some point $p \in H$. Then there exists a symmetric neighbourhood $P$ of the unit element $e$ of $H$ and a continuous map $\psi: P \times I \rightarrow H$ such that $\psi(u, 0)=u, \psi(u, 1)=e=\psi(e, t)$, for all $u \in P$ and $t \in I$.

ProOF. Let $\psi: W \times I \rightarrow H$ be a continuous map such that $\psi(w, 0)=$ $w, \psi(w, 1)=p$. By Lemma 6.1, we assume that $p \in W$. Next, let $V=p^{-1} W$ and define $\psi^{\prime}: V \times I \rightarrow H$ by $\psi^{\prime}\left(p^{-1} w, t\right)=p^{-1} \psi(w, t)$; clearly, $\psi^{\prime}$ is continuous and $\psi^{\prime}\left(p^{-1} w, 0\right)=p^{-1} w$ and $\psi\left(p^{-1} w, 1\right)=e$. Finally, let $P=V \cap V^{-1}$ and define $\psi: P \times I \rightarrow H$ by $\psi(x, t)=\left(\psi^{\prime}(e, t)\right)^{-1} \psi^{\prime}(x, t)$. It is easily seen that $P$ and $\psi$ satisfy all requirements.

Proposition 6.3. If a Hausdorff $k$-space $Z$ is $L E C$ then its cone $C Z$ is $E C$.

Proof. Clearly $C Z$ is LEC, by the Adjunction theorem on page 678 of [8], with $X=Z \times I$ ( $X$ is LEC, by Theorem II.2 of [8]), $A=Z \times\{0\}, Y=\{p\}$, 
for some $p \notin X$, and $f: A \rightarrow Y$ the constant function. (Note that the map $\pi_{1}: Z \times I \rightarrow A$, defined by $\pi_{1}(z, t)=(z, 0)$, is a retraction. Also the map $\pi_{2}: Z \times I \rightarrow I$, defined by $\pi_{2}(z, t)=t$, is a halo for $A$ in $X$.) Therefore, by Theorem 2.4 of [6], $C Z$ is EC.

EXAMPLE 6.4. The space $H$ in Lemma 5.1 is contractible to $p=(0,1 / 2)$ but it is not $p$-contractible. $H$ is also weakly locally contractible but is not locally contractible. (Note that if $h: H \times I \rightarrow H$ were a homotopy such that $h(p, t)=p$, for all $t \in I$, then, by compactness of $I$, there would exist $\delta>0$ such that $h(B(p, \delta) \times I) \subset B(p, 1 / 4)$, where $B(x, s)$ denotes the ball with center $x$ and radius $s$. This is impossible since there are no $\operatorname{arcs}$ in $B(p, 1 / 4)$ joining $p$ to points with a nonzero abscissa. This also shows that $H$ is not locally contractible.)

\section{References}

[1] C. R. Borges, 'A study of absolute extensor spaces', Pacific J. Math. 31 (1969), 609-617.

[2] _ 'Absolute extensor spaces: A correction and an answer', Pacific J. Math. 50 (1974), 29-30.

[3] _ , '(Local) mixers and (L)EC-spaces', Math. Japon. 30 (1985), 85-88.

[4] _ , 'Free topological groups', J. Austral. Math. Soc. Ser. A 23 (1977), 360-365.

[5] _ ' 'Free groups, symmetric and reduced products', J. Austral. Math. Soc. Ser. A 28 (1979), 174-178.

[6] J. Dugundji, 'Locally equiconnected spaces and absolute neighborhood retracts', Fund. Math. 57 (1965), 187-193.

[7] J. Dugundji, Topology (Allyn and Bacon, Boston, Mass., 1966).

[8] E. Dyer and S. Eilenberg, 'An adjunction theorem for locally equiconnected spaces', Pacific J. Math. 41 (1972), 669-685.

[9] R. Edwards and R. Kirby, 'Deformations of spaces and embeddings', Ann. of Math. (2) 93 (1971), 63-88.

[10] M. I. Graev, 'Free topological groups', Amer. Math. Soc. Transl. (Ser. 1) 8 (1962), 305-364.

[11] I. M. James, 'Reduced product spaces', Ann. of Math. (2) 62 (1955), 179-197.

[12] E. Katz, S. A. Morris and P. Nickolas, 'Free abelian topological groups and adjunction spaces' to appear.

[13] K. Sakai, 'A characterization of local equiconnectedness', Pacific J. Math. 111 (1984), 231-241.

[14] S. Willard, General Topology (Addison-Wesley, Reading, Mass., 1970).

\section{Department of Mathematics}

University of California

Davis, California 95616

U.S.A. 